\title{
LA SPEDIZIONE DELL'I.N.G. A KHARTOUM IN OCCASIONE DELL'ECLISSE TOTALE DI SOLE DEL $\left.25 / 2 / 1952{ }^{*}{ }^{*}\right)$
}

\author{
R. Cialdea
}

In questa prima relazione riferirò sulla organizzazione delle ricerche svolte a Khartoum durante l'eclisse totale di sole del $25 \mathrm{feb}$ braio 1952. Certamente le eclissi di sole hanno una grandissima importanza dal punto di vista geofisico, giacché permettono di approfondire le conoscenze sulle relazioni tra i fenomeni solari e quelli terrestri. Infatti, le radiazioni sia corpuscolari che elettromagnetiche del sole producono sulla terra e nell'atmosfera terrestre effetti termici, luminosi e ionizzanti, effetti che possono influire in misura più o meno grande su tutti i fenomeni geofisici della termodinamica, dell'ottica, dell'elettricità atmosferica e del magnetismo terrestre. La luna pertanto, con il suo passaggio davanti al disco solare, costituisce uno schermo che si sposta rapidamente; il sole cosi, può essere considerato come una sorgente o termica o luminosa o ionizzante più o meno oscurata. Tale rapido oscuramento può far sperare di poter separare l'influenza della radiazione solare diretta da quella di tutti gli altri fenomeni che avvengono sulla terra. Inoltre, una eclisse totale di sole si presenta come oscuramento parziale dell'atmosfera, ciò che fa sorgere altri problemi relativi ai fenomeni secondari di riemissione della radiazione diretta. Purtroppo fino ad ora le osservazioni dei fenomeni geofisici, ad eccezione delle numerose misure nel campo ionosferico, durante le eclissi ${ }^{\left({ }^{1}\right)}$ sono state sporadiche e spesso contraddittorie; naturalmente questo fatto deriva in gran parte dalla notevole influenza dei fenomeni locali meteorologici sulle osservazioni effettuate. Questa eclisse, visibile nel Sudan, in un clima, come vedremo in seguito, estremamente secco, si presentava particolarmente favorevole alle dette osservazioni. Fu deciso perciò l'impianto di un osservatorio geofisico temporaneo nella zona di totalità; per questo scopo elaborai un piano di ricerche, che, data la notevole lontananza

(*) Comunicazione presentata al Convegno dell'Associazione Geofisica Italiana, tenuto a Roma il 10-11 giugno 1952. 
della base scelta e le difficoltà dei trasporti, fu ridotto solo a quattro gruppi di osservazioni:

$1^{\circ}$ andamento della temperatura virtuale del cielo;

$2^{\circ}$ studio della luce diffusa dell'atmosfera;

$3^{\circ}$ misure delle proprietà elettriche dell'atmosfera;

$4^{0}$ misure del campo magnetico terrestre.

Si rendeva inoltre necessaria la registrazione di questi dati per un certo periodo, allo scopo di ricavare l'andamento medio diurno nella zona di totalità, per poter rilevare un eventuale effetto dell'eclisse. In tal modo si ricavava anche l'andamento diurno delle grandezze esaminate in una zona equatoriale.

Riferirò ora in particolare sui problemi connessi con dette ricerche e sulla preparazione degli apparati allestiti a questo scopo.

Temperatura virtuale del cielo. - Per lo studio dell'equilibrio termico dell'atmosfera può essere utile sia la conoscenza del raffreddamento dell'atmosfera stessa durante un'eclisse totale tra il primo, il secondo e il terzo contatto, quando cioè la radiazione diretta del sole diminuisce e si annulla, sia la conoscenza del riscaldamento dell'atmosfera stessa dopo il terzo contatto, quando cioè questa radiazione riaumenta. Una grandezza che può caratterizzare il raffreddamento ed il riscaldamento della atmosfera, è la temperatura virtuale del cielo $\left({ }^{2}\right)$. Per questo scopo possono essere adoperati metodi assoluti (per esempio piroeliometri a compensazione elettrica del tipo di Ângström) o metodi relativi (per esempio solarigrafi). Ora, data la necessità di un funzionamento continuo ed automatico dell'apparato, non potei scegliere un metodo assoluto. Scelsi allora un solarigrafo tipo Gòrczynski della Kipp e, come strumento, un galvanometro Hartmann e Braun, dotato di una serie di shunt per le diverse sensibilità.

Luce diffusa dell'atmosfera. - L'eclisse totale di sole può considerarsi come un'ombra cilindrica che investa tutta l'atmosfera e che scorra sulla terra nella zona di totalità. Ora, dato che al centro di questa ombra la illuminazione prodotta direttamente dal sole è minima, poiché la corona esterna del sole è pochissimo luminosa, l'eclisse permette lo studio della diffusione secondaria dell'aria. Infatti la brillanza dell'atmosfera nel centro dell'ombra è dovuta solo alla radiazione diffusa dalle regioni esterne all'ombra e ridiffusa da quelle 
interne. Inoltre l'illuminazione della zona d'ombra risulta del tutto anormale, dato che essa è dovuta esclusivamente ad un cilindro luminoso (le regioni esterne all'ombra) invece che ad un fascio parallelo di luce (illuminazione diretta del sole). Per questa ragione era interessante lo studio dello stato di polarizzazione di tutta la volta celeste.

Per poter eseguire tale ricerca fu necessario preparare un apparato che permettesse la misura istantanea dello stato di polarizzazione di tutta la volta celeste, poiché il fenomeno è rapidamente variabile nel tempo. Tale apparato è il nefoscopio fotografico a tre camere, che sarà più diffusamente descritto in una nota seguente; esso consiste essenzialmente in tre apparecchi folografici posti l'uno sopra l'altro su un telaio metallico (fig. 1), ciascuno dei quali fotografa una semisfera metallica con la superficie riflettente. In tal modo, di tulta la volta celeste si forma una immagine virtuale nell'interno di ciascuna sfera, immagine che

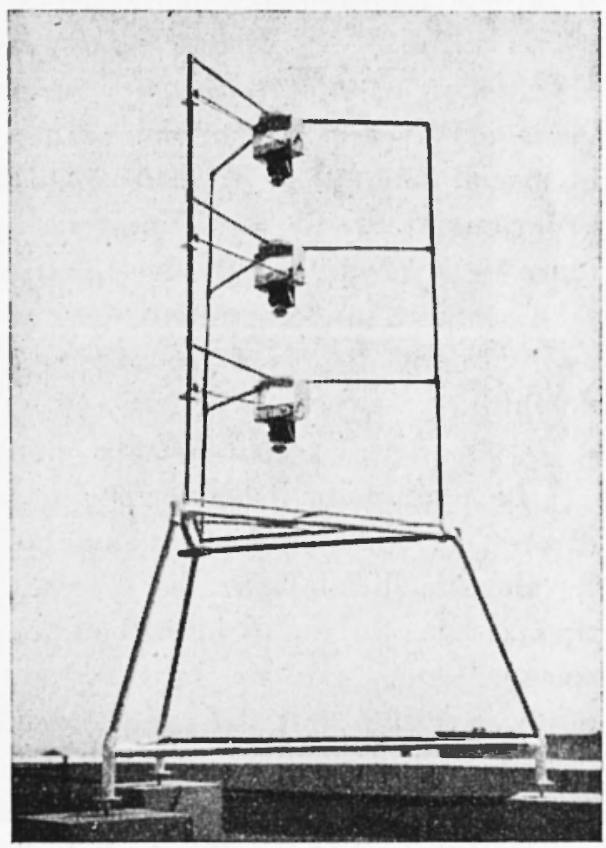

Fig. 1 viene poi ripresa dall'apparecchio fotografico corrispondente. Gli specchi e gli apparecchi (NettarZeiss con obiettivo Novar $f: 4,5$ ) sono posti in modo da mascherare il minimo angolo solido possibile. Ciascun obiettivo è dotato di un filtro di luce e di un polaroid; i piani di vibrazione (p.d.v.) di questi pola. roid sono posti a $60^{\circ}$ l'uno dall'altro. In questo modo si ottengono tre fotografie simultanee di tutta la volta celeste: dai rispettivi annerimenti di ciascuna zona si possono allora ricavare l'intensità totale della luce diffusa, il grado di polarizzazione e la posizione del p.d.v. della componente polarizzata, grandezze queste che definiscono lo stato di po- 
larizzazione. Tale apparato fu appositamente costruito nelle officine dell'I.N.G. per questa occasione.

Inoltre si adoperava anche un nefoscopio fotoelettrico $\left({ }^{3}\right)$, costituito da una semplice lente che proietta una certa zona fissa del cielo su una cellula fotoelettrica a strato di sbarramento: un polaroid rotante viene interposto tra la lente e la cellula. Uno strumento misura la corrente fotoelettrica e quindi l'intensità della luce diffusa dalla zona esaminata. Dalla misura dei massimi e dei minimi della corrente si ottiene il grado di polarizzazione.

Elettricità atmosferica. - Durante l'eclisse totale di sole inoltre viene a mancare l'azione ionizzante della radiazione, che si risente in special modo nella stratosfera. Questa diminuzione può influire sulle proprietà elettriche dell'atmosfera. Si rendeva così necessario effettuare delle misure elettriche e preparai pertanto i seguenti apparati:

$1^{\circ}$ due sonde radioattive di potenziale al polonio;

$2^{\circ}$ due dispersori tipo Elster e Geitel per la misura della conducibilità ;

$3^{\circ}$ quattro elettrometri a quadrante tipo I.N.G.

Le due sonde di potenziale dovevano essere poste a due quote diverse, in modo che la variazione del campo con l'altezza desse anche il valore medio della carica spaziale. Il supporto delle sonde era del tipo a stilo, isolato in basso con un grosso blocco di elettrina. I di. spersori erano formati da sfere metalliche del diametro di $20 \mathrm{~cm}$, poste su stili isolati. La misura della conducibilità doveva essere effettuata col metodo classico caricando ogni minuto il dispersore a 50-100 volt positivi o negativi e lasciandolo poi scaricare per semplice conducibilità elettrica. Tutti gli isolatori erano in elettrina preparata allo scopo dall'officina dell'I.N.G., che provvide inoltre alla costruzione dei porta sonde, dei dispersori e degli stili.

Magnetismo terrestre. - La variazione della ionizzazione degli alti strati dell'atmosfera poteva inoltre influire sul campo magnetico terrestre. A tale scopo si previde una stazione magnetica completa, composta di tre variometri del tipo Ruska.

Apparati accessori. - Tutti gli apparati ai quali ho accennato comportano dei galvanometri e degli elettrometri (in tutto sei strumenti), che hanno bisogno di una registrazione fotografica. Previdi allora un unico registratore fotografico a più tracce, ciò cbe permet- 
teva uno spoglio più rapido delle registrazioni ed una manovrabilità più semplice. Poiché era necessario uno scorrimento rapido $(2 \mathrm{~mm}$ al minuto, cioè $288 \mathrm{~cm}$ al giorno) si usava un forte motore a molla; occorreva poi una larga banda di carta fotografica della dimensione di $60 \mathrm{~cm}$, ciò che permetteva la registrazione di otto tracce completamente separate luna dall'altra. $F u$ cosi realizzato un registratore

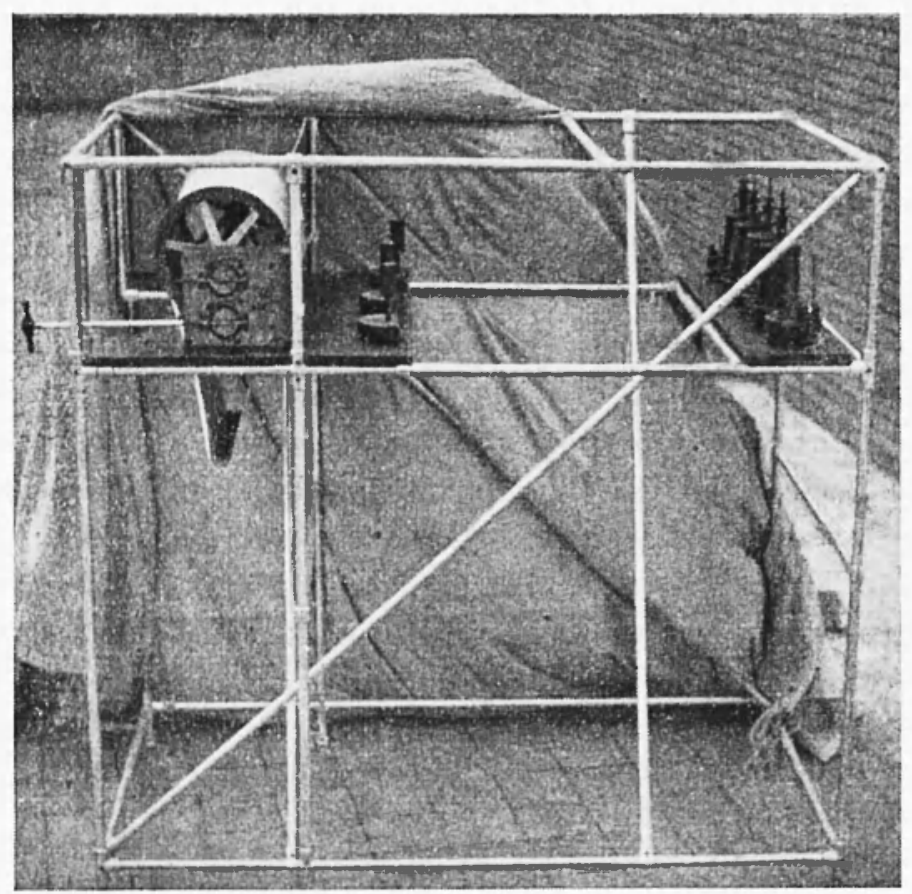

Fig. 2

fotografico costituito da una grande incastellatura di $200 \times 170 \times 90$ $\mathrm{cm}$, in tubo di alluminio, leggerissima e tutta smontabile. Un grande tamburo di $60 \times 30 \mathrm{~cm}$ era azionato a frizione da un robusto motore a doppia molla con una carica di circa 18 ore. L'incastellatura (fig. 2) formava due scompartimenti separati a tenuta di luce: uno destinato alla carta fotografica, l'altro agli strumenti ed alle lampadine elettriche necessarie per la registrazione. 11 tamburo per la carta folografica era addossato alla parete divisoria superiore dotata di una lunga fenditura orizzontale, sulla quale gli strumenti proiettavano l'immagine dei filamenti rettilinei di alcune lampadine elettriche. 'Tulto il registra- 
tore veniva poi coperto da un grande tendone nero con una finestra anteriore dotata di un foglio di celluloide rosso per l'ispezione agli strumenti ed alla registrazione; sulla parte superiore si trovavano gli isolatori in ebanite per $i$ contatti elettrici dei galvanometri e delle lampadine elettriche e gli isolatori in elettrina per $i$ contatti con gli elettrometri.

Riguardo poi alla alimentazione elettrica, furono previste delle batterie di accumulatori al piombo a sei volt.

Organizzazione generale della spedizione. - Tutti questi apparati dovevano costituire l'attrezzatura dell'osservatorio geofisico temporaneo da installarsi in una zona di totalità cioè a Klıartoum, cbe era l'unica città in vicinanza alla linea di totalità $(8-9 \mathrm{~km})$ ed al punto di massima durata dell'eclisse (circa $800 \mathrm{~km}$ ). Infatti l'eclisse totale di sole del 25 febbraio 1952 ha avuto inizio nel golfo di Guinea; la sua zona di totalità descriveva quasi un arco percorrendo tutta l'Africa centrale fino a tagliare il Sudan Anglo-Egiziano passando a pochi chilometri da Khartoum, poi, sfiorando porto Sudan traversava il mar Rosso, dove si trovava il punto di massima durata dell'eclisse, proseguiva attraversando l'Arabia, e, sfiorando Bassora attraverso l'Iran, passava nell'Asia centrale russa dove aveva termine l'eclisse.

Inoltre Khartoum presentava un clima veramente favorevole alle nostre ricerche. Riporterò qui infatti le medie mensili del mese di febbraio tolte dal bollettino del Sudan Meteorological Service:

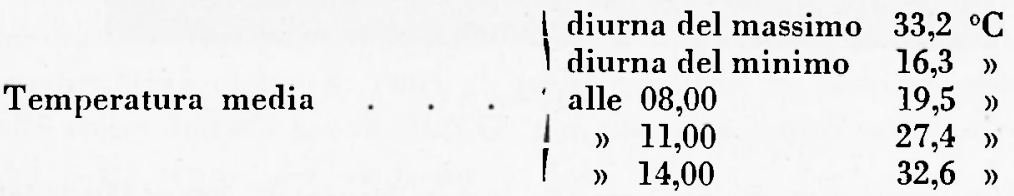

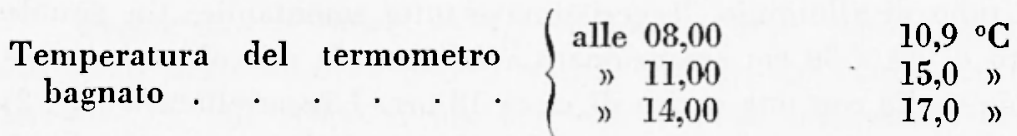

Umidità relativa . . $\quad\left\{\begin{array}{cc}\text { alle } 08,00 & 30 \% \\ \geqslant 11,00 & 21 \% \\ \prime 11,00 & 17 \%\end{array}\right.$

Piovosità media . . . . . . . . . $0 \mathrm{~mm}$ 


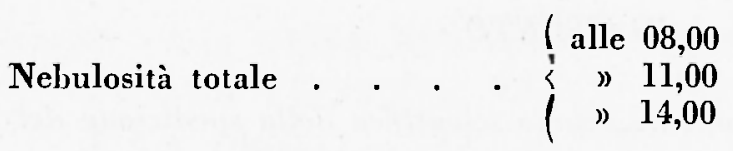

Nebulosità nubi sotto $2500 \mathrm{~m}$

Vento

Visibilità . . . . .
1,8 decimi

1,5 »

1,7 ॥

0 decimi

I $1 \%$ calmo $89 \% \quad \mathrm{l}<\mathrm{f}<3$

$\mid \begin{array}{ll}10 \% & f>4\end{array}$

$6 \%<10 \mathrm{~km}$ $99 \%<50$ "

$1 \%>50$ ")

alle 08,00

) 11,00

) 14,00
$12 \%<10 \%$

$100 \%<50$ »

( $0 \%>50 "$

$\left\{\begin{array}{r}7 \%<10 \\ 100 \% 50 \% \\ 0 \%>50 \%\end{array}\right.$

Da questo quadro si può vedere come le condizioni climatiche siano veramente ottime per le suesposte ricerche: l'umidità relativa così bassa, la nebulosità così scarsa e la piovosità nulla, facevano prevedere una buona riuscita delle osservazioni. Infatti tutti i fenomeni della termodinamica, dell'ottica e della elettricità atmosferica sono particolarmente influenzati dalla presenza del vapor d'acqua e dei fenomeni di condensazione ed evaporazione. Infine Khartoum, con la sua organizzazione di città europea, favoriva particolarmente tutta la parte logistica della spedizione. È da tener presente pure che l'Unione Astronomica Internazionale aveva scelto Khartoum come base per le osservazioni astronomiche; infatti ben 18 spedizioni di 11 Nazioni diverse furono ospitate dal Sudan Anglo-Egiziano per questa occasione. Per tali motivi quindi fu deciso di installare l'osservatorio nella zona di Khartoum.

Nella relazione seguente riferirò, insieme al dott. Piero Dominici geofisico dell'I.N.G., sull'impianto di questo osservatorio e sull'andamento generale delle ricerche effettuate.

Roma - Istituto Nazionale di Geofisica - Maggio 1952. 


\section{RIASSUNTO}

L'A. riferisce sulla organizzazione scientifica della spedizione dell'I.N.G. inviata a Khartoum per eseguire osservazioni di fenomeni geofisici in occasione dell'eclisse totale di sole del 25 febbraio 1952. In particolare si riferisce sugli apparati allestiti a questo scopo per la temperatura virtuale del cielo, per la distribuzione dello stato di polarizzazione su tutta la volta celeste, per le proprietà elettriche dell'atmosfera (potenziale e campo elettrico, carica spaziale media, conducibilità elettrica) e per il magnetismo terrestre.

\section{SUMMARY}

The author reports on the scientific organization of the I.N.G. Expedition sent to Khartoum to make geophysical observations during the total solar eclipse of February 25, 1952. Mentioned in particular various types of apparatus for measuring the virtual temperature of the sky, for the distribution of the state of polarization of the sky, for the electric properties of the atmosphere (potential and electric field, average spacial charge, electric conductivity) and for the terrestrial magnetism.

\section{BIBLIOGRAFIA}

(1) E. Mathias: Traite d’électricité atmosphérique. Paris 1924.

H. F. Johnston: Terrest. Magn. 29 (1924) p. 13.

I. P. Ault, S. J. Mauchly, R. H. Goddard: Terrest. Magn, 30 (1925) ·p. 125.

H. E. SeemanN: Frank. Jnst. J. 200 (1925) p. 629.

P. J. Nolan, C. O'Brolchain: Roy. Irish Acad. Proc. 38 (1928) p. 1.

S. Nakamura, E. Hukusima: Tohoku Imp. Univ. Sci. Reports. 19 (1930) p. 283.

N. A. Critikos: Gerlands. Beütr. z. Geophys. 50 (1937) p. 1.

M. Pierucci: N. Cimento. 16 (1939) p. 225.

J. Q. Stewart, C. D. Mac Cracken: Astrophys. J. 91 (1940) p. 51.

E. O. Hulburt: Nat. Geogr. Soc. Contrib. Techn. Pap. 2 (1942) p. 78.

M. W. Jones, A. A. Giesecke: Terr. Magn. Atmos. Elect. 49 (1944) p. 119.

O. H. Gish: Terr. Magn. Atmos. Elect. 49 (1944) p. 123.

R. Cinddfa: Ricerca Scient. e Ricostr. 15 (1945) p. 385.

J. Rouch: C. R. Acad. Sci. Paris 228 (1949) p. 1547.

(2) A. Lo Surdu: Nuovo Cimento. 5 (1913) p. 437.

(3) R. Cialdea: Ricerca Scient. e Ricostr. 17 (1947) p. 633. 